\title{
Impact of Conducting Materials on Furan- Spectral Correlation of Transformer Oil
}

\author{
Narottam Das, Senior Member, IEEE, A. Abu-Siada, Senior Member IEEE, and Syed Islam, Senior Member, \\ IEEE Department of Electrical and Computer Engineering, Curtin University, Perth, WA, Australia \\ e-mail: narottam.das@curtin.edu.au, a.abusiada@curtin.edu.au, and s.islam@curtin.edu.au
}

\begin{abstract}
This paper investigates the impact of conducting materials on the correlation of furan concentration in transformer oil and its spectral response characteristics. The presence of furan derivatives in aged-transformer oil has been one of the key indicators for solid dielectric deterioration. Identification and quantification of furan derivatives are currently performed using high performance liquid chromatography or gas chromatography-mass spectrometry based on ASTM D5837 standard. Although these techniques are well established, they need an expert personal to conduct the test and to analyse its results. Moreover, the test is relatively expensive and the analysis of oil sample may take days as most utilities sending transformer oil samples to external laboratories to be tested. Recently, it has been proven that furan concentration in transformer oil has a strong correlation to the oil spectral response. This paper is to validate this finding and to investigate the impact of conducting materials within transformer oil on this correlation. In this context, extensive experimental tests are conducted to assess the impact of various concentration of conducting materials (e.g., copper dust) dissolved in transformer oil samples of different furan contents on its absorption and transmission spectra. The measured experimental results show that; the impact of such-small amount ofdissolved -conducting materials on the oil spectral response is minimal and the previously estimated correlation can still be applied.
\end{abstract}

Index Terms - Transformer oil, Furan derivatives, Spectral response, light absorption and transmission.

\section{INTRODUCTION}

$\mathrm{P}$ ower transformers are vital links within any transmission and distribution networks. Condition monitoring and diagnostic techniques are essential to decrease the maintenance cost and improve the reliability of the equipment's or electrical assets. At present, there are several chemical and electrical diagnostic techniques applied for power transformers. Furans are the main chemical compounds that are produced as the paper insulation ages and degrades due to the thermal and electrical stresses that a transformer experiences during its operation. The paper insulation is a critical component in oil-immersed transformers as in general; transformer operational life span is equivalent to the age of the solid dielectric.

The research on identifying furans in transformer oil started over the last three-decades and reported that the thermal degradation of cellulosic insulation material within the paper insulation results in five furan compounds namely; 2furaldehyde, 5-hydroxymethyl-2-furaldehyde, 2-acetylfuran, 5-methyl-2-furaldehyde and 2-furfurol. These products dissolve in insulation oil and decreases paper and oil dielectric strength [1]. The dielectric strength of paper insulation can be accurately assessed by measuring its degree of polymerization (DP). However, it is impractical to acquire paper samples from an operating transformer especially paper samples from hotspot location that is usually located in the centre of the transformer winding. There has been a lot of research in the literature that concludes a strong correlation between the DP and furan derivatives concentrations [1]-[4]. The advantage of furan measurement compared to the DP is that the oil samples can be conveniently collected from an operating transformer without causing any damage to the transformer.

An increase in furan concentration in transformer oil corresponds to a decrease in the tensile strength and the DP of the insulation paper. Furan level in a transformer can be correlated with the paper DP, and therefore an in-service assessment of the mechanical strength of the paper insulation can be made. De Pablo reported the following relation between the furfural and DP based on viscosity $\left(\mathrm{DP}_{\mathrm{V}}\right)$ [5-6].

$$
D P_{V}=\frac{7100}{8.88+2 F A L}
$$

where, 2FAL is the 2-furfural concentration in $\mathrm{mg} / \mathrm{kg}$ of oil.

ASTM D5837 is a commonly used guideline by the industries to determine the furan derivatives concentration in the transformer oil using high performance liquid chromatography (HPLC) or gas chromatography-mass spectrometry (GC/MS) [7]-[14]. Since this method requires specific skills and expensive equipment to conduct the test and to analyse its results, most utility companies outsource the furan test to the relevant service companies.

The purpose of this paper is to measure and to analyse the influence of conducting materials (such as, copper, aluminium or any other conducting materials) on the furan concentration and its effect on the remaining life of the transformer using ultraviolet-to-visible (UV-Vis) spectroscopy. The conducting materials increase the absorption area and decrease the transmission area. Hence, the transformer life is decreased. In this context, several tests were conducted on different transformer oil samples with different level of furan concentration. A custom-designed Matlab algorithm is 
developed based on the parameters taken from the UV-Vis spectral response curve to estimate the furan concentration within the transformer oil samples.

This paper is organized as follows: Introduction is in section I, Experimental set up for measurement is in section II. Section III presents the results and discussion and finally the conclusion is in section IV.

\section{EXPERIMENTAL SET Up FOR MEASUREMENT}

For this measurement, several sets of transformer oil samples were collected from different in-service transformers with different operating conditions and the life span. Furan concentration in each set of oil sample was identified using GC/MS in accordance to ASTM D 5837 (B) [11]. Each test sample was pre-treated with acetonitrile prior to extraction for test specimen that was then arranged into GC/MS for furanic derivatives identification and quantification.

The spectral response (light absorbance or transmission) of oil samples were then measured using a laboratory-based UVVis spectroscopy. The experimental setup for the light absorbance (or transmission) measurement of transformer oil using the UV-Vis spectroscopy is shown in Fig. 1. The light source transmits the input light via an input optical fiber into a cuvette located in a cuvette holder, where the transformer oil sample is placed. This input light interacts with the transformer oil sample and the output optical fiber carries the output light from the transformer oil sample to the spectrometer which is connected to a PC for data analysis.

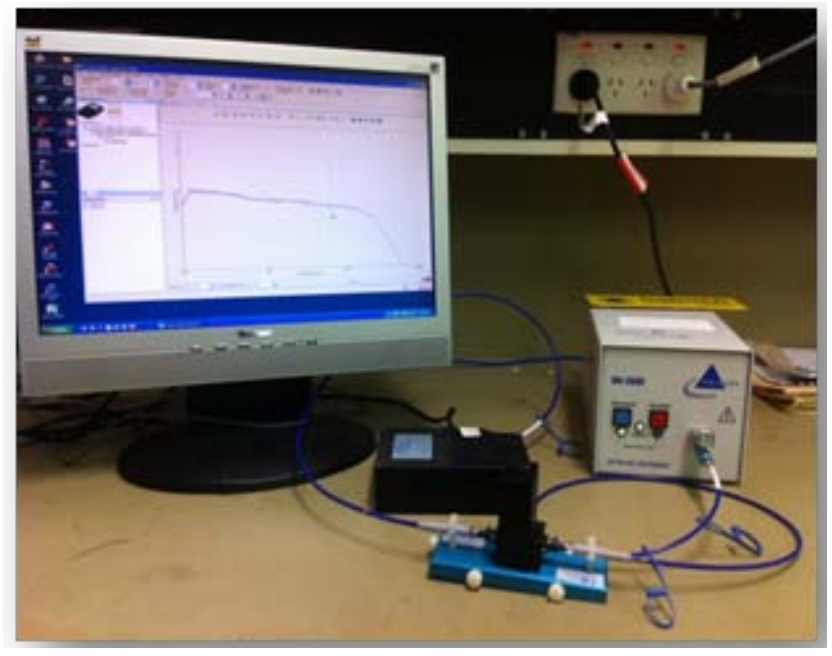

Fig. 1. Experimental setup for light absorbance (or transmission) measurement of transformer oil samples using the UV-Vis spectroscopic system.

The UV-Vis spectroscopy test procedure was developed in reference to the ASTM E 275 [14]. Furan derivatives in oil sample affect the amount of UV-Vis light absorbed or transmitted by the specimen which is calculated using the following equations.

$$
\begin{aligned}
& A_{\lambda}=-\log _{10}\left(\frac{S_{\lambda}-D_{\lambda}}{R_{\lambda}-D_{\lambda}}\right) \\
& \% T_{\lambda}=\frac{S_{\lambda}-D_{\lambda}}{R_{\lambda}-D_{\lambda}} \times 100 \%
\end{aligned}
$$

where, $A_{\lambda}$ is the light absorption, $T_{\lambda}$ is the light transmission, $S_{\lambda}$ is the sample intensity, $D_{\lambda}$ is the dark intensity, and $R_{\lambda}$ is the reference intensity at wavelength $\lambda$.

\section{RESUlTS AND DISCUSSIONS}

Fig. 2 shows the absorption spectra for various 2-FAL contents in part per million (ppm) of power transformer oil measured by the UV-Vis spectroscopy. For this test, several sets of oil samples whose furan contents were identified using GC/MS are included for the light absorption test. As can be seen in Fig. 2, the oil UV-Vis spectral response strongly correlates with its furan content [15], [16]. It is clear that the new oil with 2-FAL of $0 \mathrm{ppm}$ has the lowest spectral response area compared to the samples with higher level of furan content. Also it is observable that the new oil with 2-FAL of 0 ppm has the shortest bandwidth and lowest absorbance peak. As the 2-FAL concentration increases, the spectral response bandwidth is becoming wider and maximum absorbance peak is becoming higher. The spectral response has displayed spikes characteristic at a wavelength around $350 \mathrm{~nm}$ when furans content level increases in transformer oil. It is worth to mention that all the maximum peaks appear within the range of $260 \mathrm{~nm}$ to $280 \mathrm{~nm}$ and the zero crossing points with the $\mathrm{x}$ axis (wavelength) are not overlapped for different samples with different furan concentration levels [15], [16].

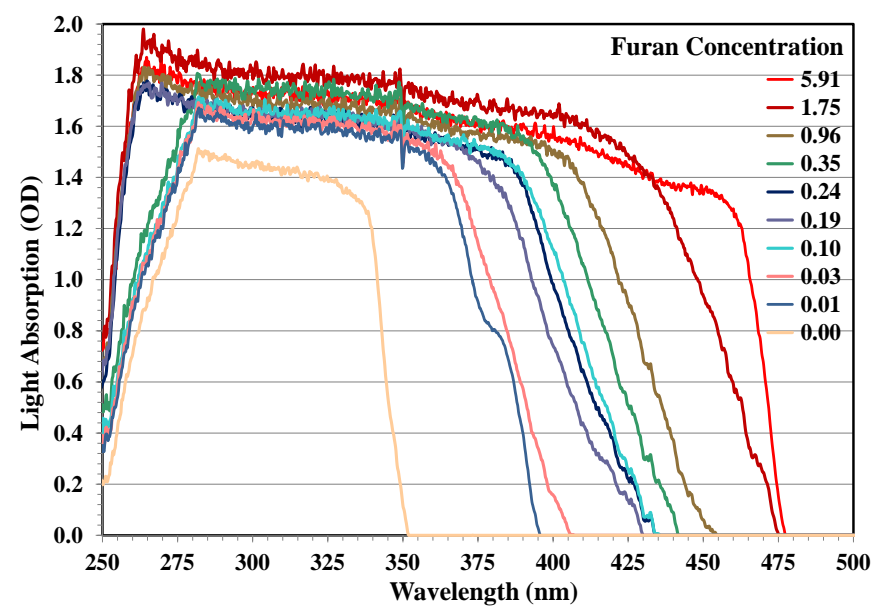

Fig. 2. Light absorption spectra for different furan concentration of power transformer oil measured by the UV-Vis spectroscopy.

Fig. 3 shows the spectral absorbance response entire area versus the furan concentration for various transformer oil samples. The figure shows clearly that there is strong correlation between the spectral response area and furan concentration in transformer oil samples which validate the previously published results in [15], [16]. A mathematical 
correlation between the spectral response area and furan concentration of transformer oil can be estimated using the fitted curve shown in Fig. 3.

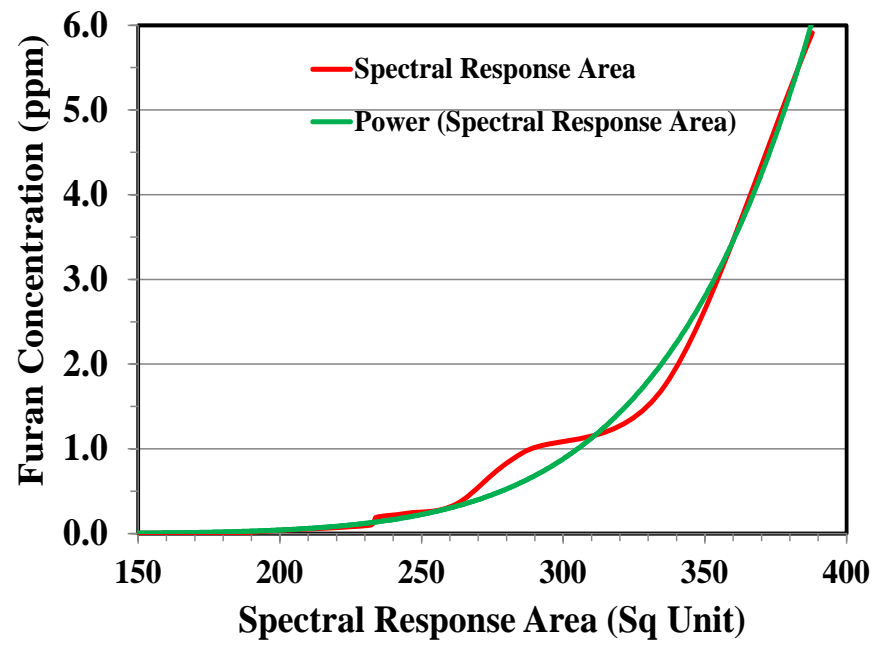

Fig. 3. Spectral response area versus the furan concentration characteristics of power transformer oil measured by the UV-Vis spectroscopy.

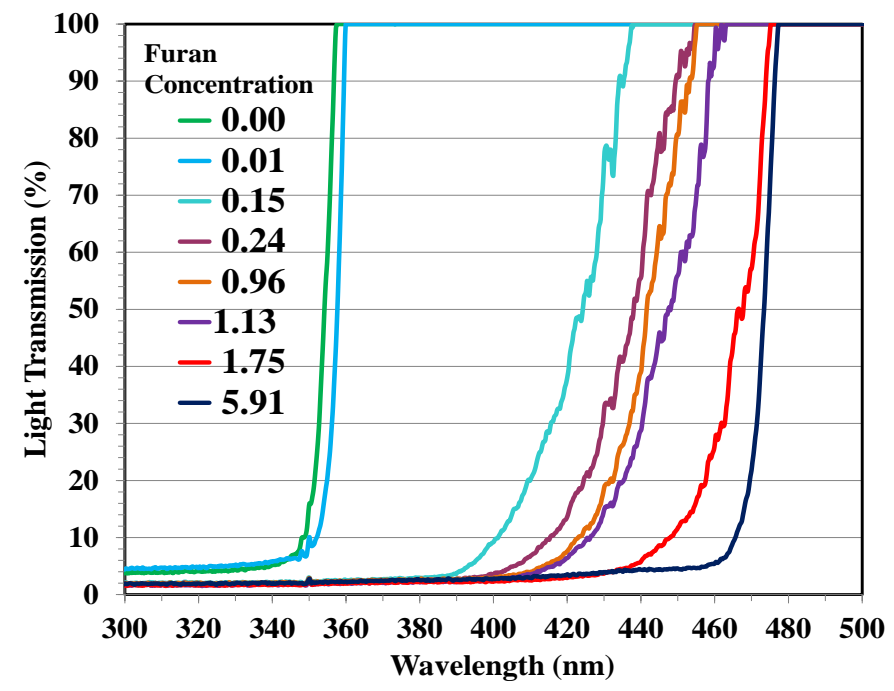

Fig. 4. Light transmission spectra for various furan concentration of power transformer oil measured by the UV-Vis spectroscopy.

Fig. 4 shows the light transmission spectra for various 2FAL contents (in ppm) of transformer oil measured by the UV-Vis spectroscopy. This figure shows that new oil with 2FAL of 0 ppm has the lowest threshold wavelength whereas samples with higher level of furan concentration has the highest threshold wavelength.

Fig. 5 shows the threshold wavelength versus furan concentration of transformer oil samples, which is obtained from the light transmission spectra shown in Fig. 4. Fig. 5 shows clearly that there is strong correlation between the threshold wavelength and furan concentration of transformer oil samples.

The conducting materials (e.g., copper dust) are mixed into the transformer oil samples with pre-known furan concentration and the light absorption and transmission through the mixture are immediately measured after adding the copper dust to the oil samples. The measured results show that the spectral response area increased for the light absorption spectra and decreased for the light transmission spectra.

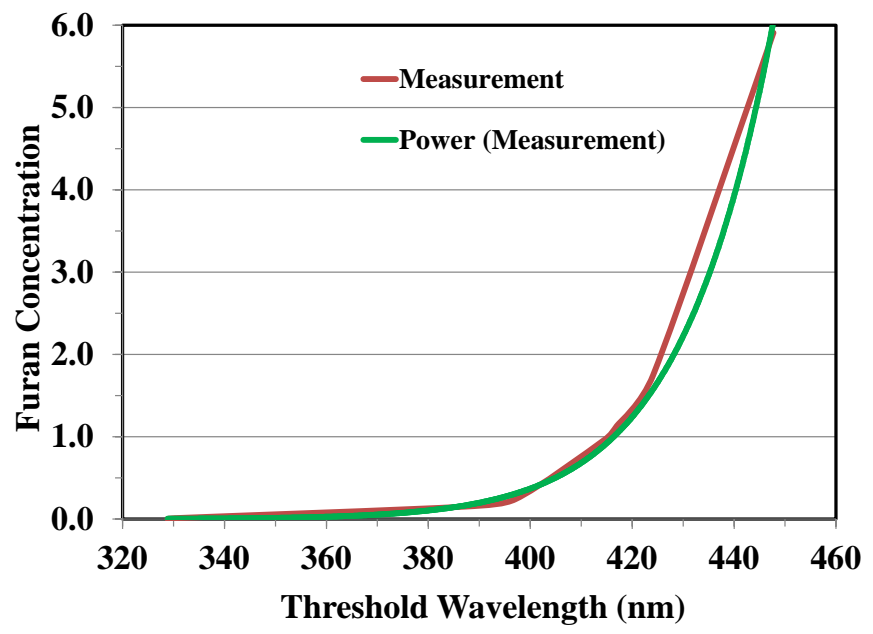

Fig. 5. Thereshold wavelength versus the furan concentration characteristics of transformer oil samples measured by the UV-Vis spectroscopy.

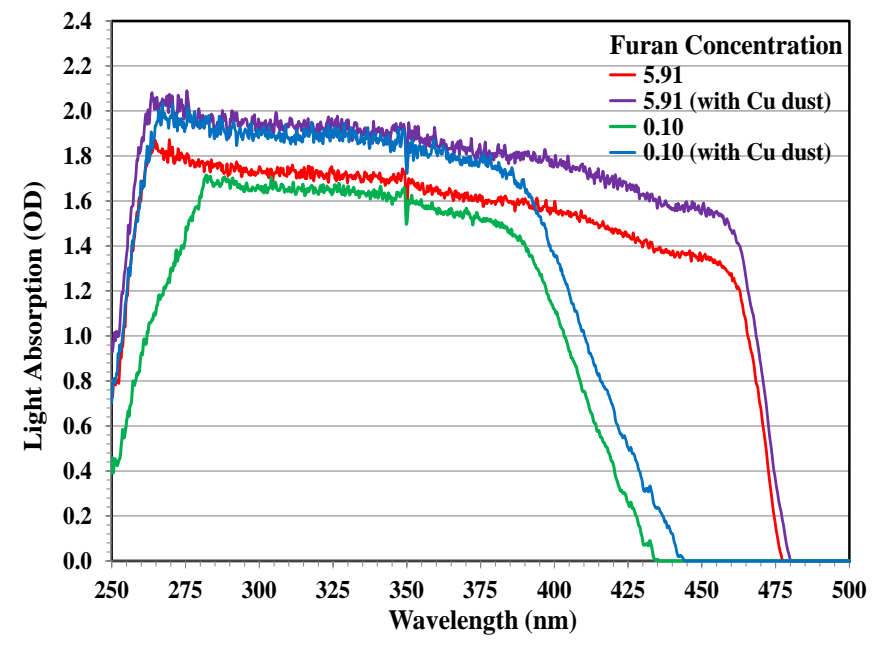

Fig. 6. Light absorption spectra of 2 different oil samples with and without copper dust with different furan concentrations measured by the UV-Vis spectroscopy.

Fig. 6 shows the light absorption spectra for 2 different oil samples with different furan concentrations (without and with the copper dust). It is clearly observed that when the conducting materials is added to oil samples the spectral response area is increased due to the higher amount of light absorption by the higher contamination within oil samples and hence the spectral response area is increased. The spectral response area for both studied samples is increased by about $15 \sim 20 \%$ and the spectral bandwidth is increased by about 5 10nm.

Fig. 7 shows the light transmission spectra for 2 different oil samples with and without the copper dust. It can be observed that when the conducting material is added to the oil samples, the light transmission is reduced and spectral response area is reduced accordingly. For both studied 
samples, area is decreased by about $10 \sim 20 \%$ and the threshold wavelength is increased by about $10 \sim 15 \mathrm{~nm}$.

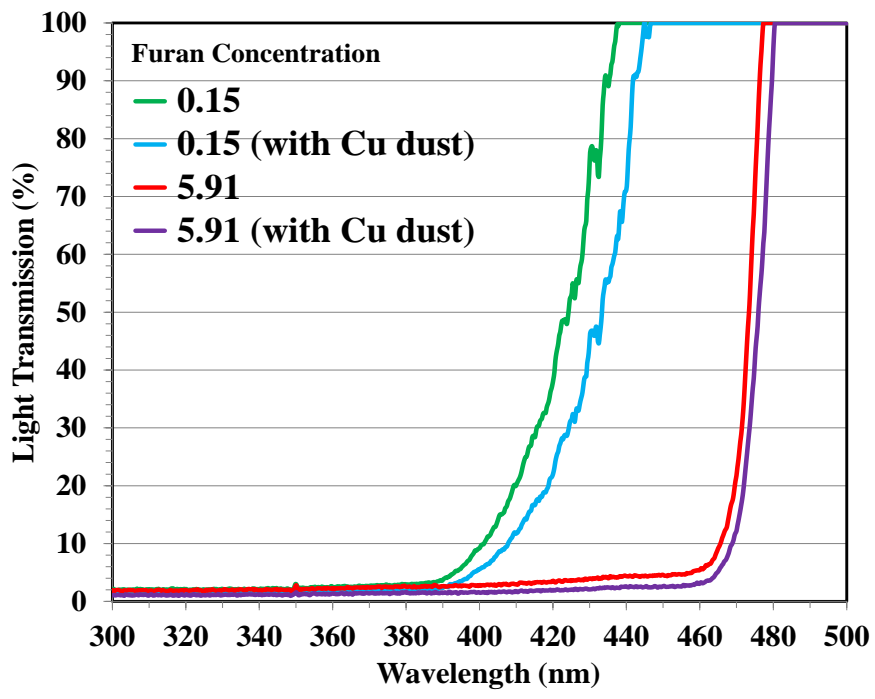

Fig. 7. Light transmission spectra for 2 different oil samples with and without the copper dust with different furan concentrations measured by the UV-Vis spectroscopy.

It is worth to mention that the above spectral responses were measured directly after adding the conducting material to the oil samples. When the spectral response was measured for the same oil samples after one hour, the response was found to be identical to the original spectral response of the oil sample without any added conducting material. This is attributed to the fact that the conducting materials with heavy specific weight will settle down at the bottom of the cuvette and its impact on the spectral response will be minimal.

\section{CONCLUSION}

This paper validates the correlation of furan content in transformer oil and its spectral response and investigates the impact of conducting materials within the transformer oil samples on this correlation. The measured results show that the spectral response area increases with the existance of conducting materials in transformer oil for light absorption. On the other hand, the spectral response area decreases for light transmission with the existance of conducting materails within the transformer oil sample. The light transmission spectra clearly identifies a unique thereshold value of each light transmission characteristic for different oil samples which proves the strong correlation of the furan contents and oil specral response.

\section{ACKNOWLEDGEMENT}

This research is supported by the Faculty of Science and Enginering, Curtin University, and the Coperative Research Centre for Infrastructure and Engineering Asset Management (CIEAM), Australia. The authors would like to acknowlwdge Western Power for providing transformwer oil sampls for this experiment.

\section{REFERENCES}

[1] L. Cheim, D. Platts, T. Prevost, and S. Xu, "Furan analysis for liquid power transformers," IEEE Electr. Insul. Mag., vol. 28, no. 2, pp. 8-21, Mar./Apr. 2012.

[2] I. Hohlein and A. J. Kachler, "Aging of cellulose at transformer service temperatures. Part 2. Influence of moisture and temperature on degree of polymerization and formation of furanic compounds in freebreathing systems,” IEEE Elect. Insul. Mag., vol. 21, no. 5, pp. 20-24, Sep./Oct. 2005.

[3] J. R. Sans, K. M. Bilgin, and J. J. Kelly, "Large-scale survey of furanic compounds in operating transformers and implications for estimating service life," in Proc. IEEE Int. Symp. Conf. Rec. Elect. Insul., 1998, vol. 2, pp. 543-553.

[4] P. Thomas, A. K. Shukla, and R. Raghuveer, "Ageing studies on paper and oil to assess the condition of solid insulation used in power transformers," in Proc. IEEE 7th Int. Conf. Solid Dielectr., 2001, pp. 69-72.

[5] A. Abu-Siada "Correlation of furan concentration and spectral response of transformer oil-using expert systems," IET Sci. Meas. Technol., vol. 5, Issue 5, pp. 183-188, 2011.

[6] M. Arshad: "Remnant life estimation model using fuzzy logic for power transformer asset management”. PhD Dissertation, Curtin University of Technology, 2005.

[7] M. Wang, A. J. Vandermaar, and K. D. Srivastava, "Review of condition assessment of power transformers in service," Electrical Insulation Magazine, IEEE, vol. 18, pp. 12-25, 2002.

[8] P. J. Baird, H. Herman, G. C. Stevens, and P. N. Jarman, "Spectroscopic measurement and analysis of water and oil in transformer insulating paper,” IEEE Trans. Dielectr. Electr. Insul., vol. 13, no. 2, pp. 293-308, Apr. 2006.

[9] J. Scheirs, G. Camino, M.Avidano, and W. Tumiatti, "Origin of furanic compounds in thermal degradation of cellulosic insulating paper," $J$. Appl. Polymer Sci., vol. 69, pp. 2541-2547, 1998.

[10] F. Shafizadeh and Y. Z. Lai, "Thermal degradation of 1, 6-Anhydro-BD-glucopyranose,” in J. Org. Chem., May 24, 1971-1972, vol. 37, pp. 278-284.

[11] Unused Hydrocarbon-Based Insulating Liquids-Test Methods for Evaluating the Oxidation Stability, IEC 61125, 1992, 1st ed., I. T. C. No.10.

[12] Standard Practices for Sampling Electrical Insulating Liquids, Standard D 923-07, ASTM, 2007.

[13] Standard Test Method for Furanic Compounds in Electrical Insulating Liquids by High-Performance Liquid Chromatography, Standard D 583-05, ASTM, 2005.

[14] Standard practice for describing and measuring performance of ultraviolet, visible, and near-infrared spectrophotometers," in ASTM E 275-01. Philadelphia, PA: ASTM, 2001, vol. 03.06, pp. 72-81.

[15] A. Abu-Siada, S. P. Lai, and S. M. Islam, "A Novel Fuzzy-Logic Approach for Furan Estimation in Transformer Oil," IEEE Trans. Power Del., vol. 27, no. 2, pp. 469-474, Apr. 2012.

[16] N. Das, A. Abu-Siada, and S. M. Islam, "New approach to estimate furan contents in transformer oil using spectroscopic analysis," in Proc. of the Australasian Universities Power Engineering Conference 2012 (AUPEC2012), Sept. 26-29, 2012, Denpasar-Bali, Indonesia. Paper P009. 